\section{Bodybuilding für Frühgeborene}

Pro Tag werden in Deutschland ca. 2.000 Kinder geboren, knapp 10\% davon sind Frühchen. Finnen fanden nun heraus, dass Muskelfitness, -kraft und Selbsteinschätzung ihrer sportlichen Fähigkeiten bei ehemaligen Frühgeborenen im Alter von 23 Jahren geringer ausfallen als bei Reifgeborenen. Dabei ist das Potenzial der Herz-Kreislauf-Funktion in beiden Gruppen gleich. Also, was empfiehlt der Neonatologe, Neuropädiater und Sportmediziner Frühgeborenen, wenn sie heranwachsen? Wohldosiertes Muskeltraining - gerne auch im Sportstudio unter fachkundiger Anleitung. Auch das Selbstwertgefühl würde profitieren!

Tikanmäki M et al. Pediatrics 2016;137:1-10

\section{Leukämierisiko nach IVF \& Co. erhöht}

Seit über 30 Jahren stehen die Techniken der assistierten Reproduktion zur Verfügung. Doch was wissen wir über das Langzeitschicksal der Kinder? In einer aktuellen Arbeit wurden alle zwischen 1984 und 2011 in Norwegen geborenen Kinder nachverfolgt. Von 1.628.658 Kindern waren 25.782 „künstlich“ befruchtet entstanden. Insgesamt wurde im Verlauf bei 4.554 Kindern Krebs diagnostiziert, davon nach assistierter Reproduktion in 51 Fällen. Somit war das Gesamt-Malignomrisiko vergleichbar. Aber: Es fand sich ein erhöhtes Risiko für Leukämie und Hodgkin-Lymphome in der Gruppe nach künstlicher Befruchtung - egal, ob nun durch konventionelle IVF oder nach intrazytoplasmatischer Spermieninjektion. Eine Botschaft, die beunruhigt: Kennen wir die Folgen unserer technischen Möglichkeiten?

Reigstad MM et al. Pediatrics

2016;137:e20152061

\section{Frühlingskinder: Höchstes MS-Risiko}

Ob jemand an MS erkrankt, hängt auch von seinem Geburtszeitpunkt ab. Wissenschaftler aus Oxford analysierten Geburtsdaten von über 21.000 MS-Patienten, die zwischen 1938 und 1980 geboren wurden. Nach ihren Berechnungen ist bei Briten die MS-Rate um 17\% höher, wenn sie im April statt im November geboren wurden, für die Jahrgänge 1965 bis 1980 liegt der Unterschied sogar bei $24 \%$. Jahreszeitlich schwankende Vitamin-D-Spiegel oder winterliche Infekte der Mutter werden als Ursachen diskutiert.

JAMA Neurol 2016; epub June 27, 2016.

\title{
Kognitiv eingeschränkte Pflegebedürftige: Welche Assessment-Skalen eignen sich?
}

\author{
Derzeit werden zahlreiche Interventionen entwickelt, die pflegende Ange- \\ hörige von kognitiv eingeschränkten Menschen unterstützen sollen. Entspre- \\ chende Interventionsstudien erfassen bislang jedoch selten die Wirkung auf \\ den Pflegebedürftigen. Daher hat eine deutsche Arbeitsgruppe eine Empfeh- \\ lung von Endpunkten und Assessment-Instrumenten aufseiten der kognitiv \\ eingeschränkten Pflegebedürftigen in derartigen Studien erarbeitet.
}

In dem Projekt bewerteten 14 Experten 16 Instrumente aus fünf Dimensionen im Hinblick auf deren Anwendbarkeit in Studien mit pflegenden Angehörigen kognitiv eingeschränkter Personen. Die Experten hatten sehr unterschiedliche Vorerfahrungen mit den einzelnen Assessments und beurteilten die Instrumente teilweise sehr heterogen. Für „herausforderndes Verhalten" stuften sie die RMBPC (Revised Memory and Behavior Problem Checklist) und das NPI-Q (NeuroPsychiatric Inventory Questionnaire) als gut geeignet ein. Bei „depressive Symptomatik" schnitt die GDS (Geriatric Depression Scale) insgesamt am besten $a b$, wobei sie nur bei leichten kognitiven Einschränkungen eingesetzt werden sollte. Bei mittelgradiger kognitiver Einschränkung eignen sich die MADRS (Montgomery-Asberg Depression Scale) oder die CDS (Cornell Scale for Depression in Dementia) besser. Für Personen mit schweren kognitiven Einschränkungen gibt es keine Empfehlungen.

Von den überprüften Instrumenten zur „Lebensqualität" bewerteten die Experten die QoL-AD (Quality of Life in Alzheimer's Disease) insgesamt am günstigsten. Alle sollten jedoch nur bei leicht kognitiv eingeschränkten Personen zum Einsatz kommen. Für die „Kognition“ wurden alle Instrumente, die MMSE (Mini Mental State Examination), die ADAS-Cog (Alzheimer's Disease Assessment Scale - Cognitive Subscale), die
DemTect (Demenz-Detektion) und das MoCA (Montreal Cognitive Assessment), für leicht bis mittelgradig kognitiv eingeschränkte Personen als gut geeignet bewertet. Bei schweren kognitiven Einschränkungen empfehlen die Experten am ehesten den MMSE. Unter den Instrumenten zur Erfassung der Alltagsfunktionen schnitten das Katz ADL Assessment und die Lawton IADL-Skala am besten ab. Wie ein entsprechender Vergleich ergab, stimmte eine Reihe dieser Einschätzungen mit dem europäischen INTERDEM-Konsensuspapier (Early detection and timely INTERvention in DEMentia) überein.

Nach Analyse der Autoren besteht in den Bereichen herausforderndes Verhalten und Lebensqualität besonderer Bedarf an deutschsprachigen Validierungsstudien zu international gebräuchlichen Instrumenten. Zudem sollten sich ihres Erachtens künftige Arbeiten verstärkt mit der Entwicklung von Instrumenten für kognitiv schwer eingeschränkte Personen befassen.

(pe)

Albrecht $D$, et al. Interventionsstudien zur Unterstützung häuslicher Pflege - Empfehlungen zu Endpunkten und Assessment-Instrumenten bei kognitiv eingeschränk-

ten Pflegebedürftigen.

Z Gerontol Geriat.

Published online:

24 June 2016
Im Bereich herausforderndes Verhalten besteht besonderer Bedarf an deutschsprachigen Validierungsstudien.

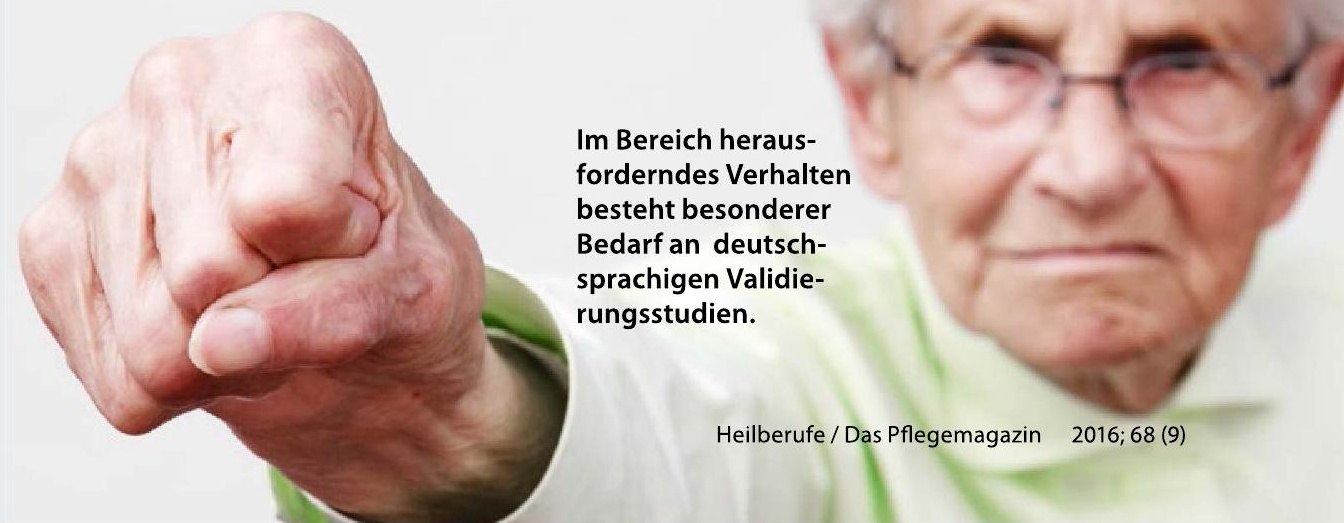

